\title{
Strong Differential Sandwich Results for Frasin Operator
}

\author{
Abbas Kareem Wanas ${ }^{1}$ and B. A. Frasin ${ }^{2}$ \\ ${ }^{1}$ Department of Mathematics, College of Science, University of Al-Qadisiyah, Iraq \\ e-mail: abbas.kareem.w@qu.edu.iq \\ ${ }^{2}$ Department of Mathematics, Faculty of Science, Al al-Bayt University, Mafraq, Jordan \\ e-mail: bafrasin@yahoo.com
}

\begin{abstract}
In this paper, we define two new classes of analytic functions involving strong differential subordinations and superordination associated with Frasin operator. Further, we study some important properties of these classes.
\end{abstract}

\section{Introduction}

Let $U$ denote the open unit disk of the complex plane $U=\{z \in \mathbb{C}:|z|<1\}$, $\bar{U}=\{z \in \mathbb{C}:|z| \leq 1\}$ be the closed unit disk of the complex plane and let $\mathcal{H}(U \times \bar{U})$ be the class of analytic functions in $U \times \bar{U}$. For a positive integer $n$ and $a \in \mathbb{C}$, let

$$
\begin{array}{r}
\mathcal{H}[a, n, \zeta]=\left\{f \in \mathcal{H}(U \times \bar{U}): f(z, \zeta)=a+a_{n}(\zeta) z^{n}+a_{n+1}(\zeta) z^{n+1}+\ldots,\right. \\
z \in U, \zeta \in \bar{U}\},
\end{array}
$$

where $a_{j}(\zeta)$ are holomorphic functions in $\bar{U}$ for $j \geq n$.

Let $\mathcal{A}_{\zeta}$ indicate the class of functions of the form:

Received: October 16, 2019; Accepted: December 13, 2019

2010 Mathematics Subject Classification: 30C45.

Keywords and phrases: strong subordinations, strong superordinations, convex function, best dominant, best subordinant.

Copyright () 2020 Abbas Kareem Wanas and B. A. Frasin. This is an open access article distributed under the Creative Commons Attribution License, which permits unrestricted use, distribution, and reproduction in any medium, provided the original work is properly cited. 


$$
f(z, \zeta)=z+\sum_{k=2}^{\infty} a_{k}(\zeta) z^{k}, \quad(z \in U, \zeta \in \bar{U})
$$

which are analytic in $U \times \bar{U}$ and $a_{k}(\zeta)$ are holomorphic functions in $\bar{U}$ for $k \geq 2$.

Definition 1.1 [4]. Denote by $Q_{\zeta}$ the set of functions that are analytic and injective on $\bar{U} \times \bar{U} \backslash E(f, \zeta)$, where

$$
E(f, \zeta)=\left\{r \in \partial U: \lim _{z \rightarrow r} f(z, \zeta)=\infty\right\}
$$

and are such that $f_{z}^{\prime}(r, \zeta) \neq 0$ for $r \in \partial U \times \bar{U} \backslash E(f, \zeta)$. The subclass of $Q_{\zeta}$ for which $f(0, \zeta)=a$ is denoted by $Q_{\zeta}(a)$.

Definition 1.2 [5]. Let $f(z, \zeta), F(z, \zeta)$ analytic in $U \times \bar{U}$. The function $f(z, \zeta)$ is said to be strongly subordinate to $F(z, \zeta)$ if there exists a function $w$ analytic in $U$ with $w(0)=0$ and $|w(z)|<1(z \in U)$ such that $f(z, \zeta)=F(w(z), \zeta)$ for all $\zeta \in \bar{U}$. In such a case we write $f(z, \zeta) \prec \prec F(z, \zeta), z \in U, \zeta \in \bar{U}$.

Remark 1.1 [5].

(1) Since $f(z, \zeta)$ is analytic in $U \times \bar{U}$, for all $\zeta \in \bar{U}$ and univalent in $U$, for all $\zeta \in \bar{U}$, Definition 1.2 is equivalent to $f(0, \zeta)=F(0, \zeta)$ for all $\zeta \in \bar{U}$ and $f(U \times \bar{U}) \subset F(U \times \bar{U})$.

(2) If $f(z, \zeta)=f(z)$ and $F(z, \zeta)=F(z)$, the strong subordination becomes the usual notion of subordination.

If $f(z, \zeta)$ strongly subordinate to $F(z, \zeta)$, then $F(z, \zeta)$ strongly superordinate to $f(z, \zeta)$.

Lemma 1.1 [3]. Let $h(z, \zeta)$ be a univalent with $h(0, \zeta)=a$ for every $\zeta \in \bar{U}$ and let $\mu \in \mathbb{C} \backslash\{0\}$ with $\operatorname{Re}(\mu) \geq 0$. If $p \in \mathcal{H}[a, 1, \zeta]$ and

$$
p(z, \zeta)+\frac{1}{\mu} z p_{z}^{\prime}(z, \zeta) \prec \prec h(z, \zeta), \quad(z \in U, \zeta \in \bar{U}),
$$


then

$$
p(z, \zeta) \prec \prec q(z, \zeta) \prec \prec h(z, \zeta), \quad(z \in U, \zeta \in \bar{U}),
$$

where $q(z, \zeta)=\mu z^{-\mu} \int_{0}^{z} h(t, \zeta) t^{\mu-1} d t$ is convex and it is the best dominant of (1.2).

Lemma 1.2 [4]. Let $h(z, \zeta)$ be a convex with $h(0, \zeta)=$ a for every $\zeta \in \bar{U}$ and let $\mu \in \mathbb{C} \backslash\{0\}$ with $\operatorname{Re}(\mu) \geq 0$. If $p \in \mathcal{H}[a, 1, \zeta] \cap Q_{\zeta}, \quad p(z, \zeta)+\frac{1}{\mu} z p_{z}^{\prime}(z, \zeta)$ is univalent in $U \times \bar{U}$ and

$$
h(z, \zeta) \prec \prec p(z, \zeta)+\frac{1}{\mu} z p_{z}^{\prime}(z, \zeta), \quad(z \in U, \zeta \in \bar{U}),
$$

then

$$
q(z, \zeta) \prec \prec p(z, \zeta), \quad(z \in U, \zeta \in \bar{U})
$$

where $q(z, \zeta)=\mu z^{-\mu} \int_{0}^{z} h(t, \zeta) t^{\mu-1} d t$ is convex and it is the best subordinant of (1.3).

For $f \in \mathcal{A}_{\zeta}, m \in \mathbb{N}, \delta, j \in \mathbb{N}_{0}=\mathbb{N} \cup\{0\}, 0 \leq \tau \leq 1$. Frasin operator [2] $D_{m, \tau}^{\delta}$ : $\mathcal{A}_{\zeta} \rightarrow \mathcal{A}_{\zeta}$ (see [7]) is defined by

$$
D_{m, \tau}^{\delta} f(z, \zeta)=z+\sum_{k=2}^{\infty}\left(1+(k-1) \sum_{j=1}^{m}\left(\begin{array}{c}
m \\
j
\end{array}\right)(-1)^{j+1} \tau^{j}\right)^{\delta} a_{k}(\zeta) z^{k}, \quad(z \in U, \zeta \in \bar{U})
$$

It is readily verified from (1.4) that

$$
C_{j}^{m}(\tau) z\left(D_{m, \tau}^{\delta} f(z, \zeta)\right)_{z}^{\prime}=D_{m, \tau}^{\delta+1} f(z, \zeta)-\left(1-C_{j}^{m}(\tau)\right) D_{m, \tau}^{\delta} f(z, \zeta),
$$

where $C_{j}^{m}(\tau)=\sum_{j=1}^{m}\left(\begin{array}{c}m \\ j\end{array}\right)(-1)^{j+1} \tau^{j}$

Special cases of this operator include the generalized Sălăgean operator [1] and the Sălăgean differential operator [6]. 


\section{Main Results}

Definition 2.1. Let $\psi(z, \zeta)$ be an analytic function in $U \times \bar{U}$ with $\psi(0, \zeta)=1$ for every $\zeta \in \bar{U}$ and $\lambda>0, m \in \mathbb{N}, \delta, j \in \mathbb{N}_{0}, 0 \leq \tau \leq 1$. A function $f \in \mathcal{A}_{\zeta}$ is said to be in the class $\mathcal{M}(\lambda, \delta, m, j, \tau ; \psi)$ if it satisfies the strong differential subordination

$$
\frac{1}{z}\left[\left(1-\frac{\lambda}{C_{j}^{m}(\tau)}\right) D_{m, \tau}^{\delta} f(z, \zeta)+\frac{\lambda}{C_{j}^{m}(\tau)} D_{m, \tau}^{\delta+1} f(z, \zeta)\right] \prec \prec \psi(z, \zeta), \quad(z \in U, \zeta \in \bar{U}) .
$$

A function $f \in \mathcal{A}_{\zeta}$ is said to be in the class $\mathcal{N}(\lambda, \delta, m, j, \tau ; \psi)$ if it satisfies the strong differential superordination

$$
\psi(z, \zeta) \prec \prec \frac{1}{z}\left[\left(1-\frac{\lambda}{C_{j}^{m}(\tau)}\right) D_{m, \tau}^{\delta} f(z, \zeta)+\frac{\lambda}{C_{j}^{m}(\tau)} D_{m, \tau}^{\delta+1} f(z, \zeta)\right], \quad(z \in U, \zeta \in \bar{U}) .
$$

Theorem 2.1. Let $\psi(z, \zeta)$ be a convex function in $U \times \bar{U}$ with $\psi(0, \zeta)=1$ for every $\zeta \in \bar{U}$ and $\lambda>0$. If $\mathcal{M}(\lambda, \delta, m, j, \tau ; \psi)$, then there exists a convex function $q(z, \zeta)$ such that $q(z, \zeta) \prec \prec \psi(z, \zeta)$ and $f \in \mathcal{M}(0, \delta, m, j, \tau ; q)$.

Proof. Suppose that

$$
\begin{aligned}
p(z, \zeta) & =\frac{D_{m, \tau}^{\delta} f(z, \zeta)}{z} \\
& =1+\sum_{k=2}^{\infty}\left(1+(k-1) \sum_{j=1}^{m}\left(\begin{array}{c}
m \\
j
\end{array}\right)(-1)^{j+1} \tau^{j}\right)^{\delta} a_{k}(\zeta) z^{k-1}, \quad(z \in U, \zeta \in \bar{U}) .
\end{aligned}
$$

Then, $p \in \mathcal{H}[1,1, \zeta]$.

Since $f \in \mathcal{M}(\lambda, \delta, m, j, \tau ; \psi)$, then we have

$$
\frac{1}{z}\left[\left(1-\frac{\lambda}{C_{j}^{m}(\tau)}\right) D_{m, \tau}^{\delta} f(z, \zeta)+\frac{\lambda}{C_{j}^{m}(\tau)} D_{m, \tau}^{\delta+1} f(z, \zeta)\right] \prec \prec \psi(z, \zeta) .
$$


From (2.1) and (2.2), we get

$$
\frac{1}{z}\left[\left(1-\frac{\lambda}{C_{j}^{m}(\tau)}\right) D_{m, \tau}^{\delta} f(z, \zeta)+\frac{\lambda}{C_{j}^{m}(\tau)} D_{m, \tau}^{\delta+1} f(z, \zeta)\right]=p(z, \zeta)+\lambda z p_{z}^{\prime}(z, \zeta) \prec \prec \psi(z, \zeta) .
$$

An application of Lemma 1.1 with $\mu=\frac{1}{\lambda}$, yields

$$
p(z, \zeta) \prec \prec q(z, \zeta) \prec \prec \psi(z, \zeta)
$$

By using (2.1), we obtain

$$
\frac{D_{m, \tau}^{\delta} f(z, \zeta)}{z} \prec \prec q(z, \zeta) \prec \prec \psi(z, \zeta),
$$

where

$$
q(z, \zeta)=\frac{1}{\lambda} z^{-\frac{1}{\lambda}} \int_{0}^{z} \psi(t, \zeta) t^{\frac{1}{\lambda}-1} d t
$$

is convex and it is the best dominant.

Theorem 2.2. Let $\psi(z, \zeta)$ be a convex function in $U \times \bar{U}$ with $\psi(0, \zeta)=1$ for every $\zeta \in \bar{U}$ and $\lambda>0$. If $f \in \mathcal{N}(\lambda, \delta, m, j, \tau ; \psi), \frac{D_{m, \tau}^{\delta} f(z, \zeta)}{z} \in \mathcal{H}[1,1, \zeta] \cap Q_{\zeta}$ and

$$
\frac{1}{z}\left[\left(1-\frac{\lambda}{C_{j}^{m}(\tau)}\right) D_{m, \tau}^{\delta} f(z, \zeta)+\frac{\lambda}{C_{j}^{m}(\tau)} D_{m, \tau}^{\delta+1} f(z, \zeta)\right]
$$

is univalent in $U \times \bar{U}$, then there exists a convex function $q(z, \zeta)$ such that $f \in \mathcal{N}(0, \delta, m, j, \tau ; q)$.

Proof. Suppose that the function $p(z, \zeta)$ be defined by (2.1). It is evident that $p \in \mathcal{H}[1,1, \zeta] \cap Q_{\zeta}$. After a short calculation and considering $f \in \mathcal{N}(\lambda, \delta, m, j, \tau ; \psi)$, we can conclude that

$$
\psi(z, \zeta) \prec \prec p(z, \zeta)+\lambda z p_{z}^{\prime}(z, \zeta)
$$


An application of Lemma 1.2 with $\mu=\frac{1}{\lambda}$, yields

$$
q(z, \zeta) \prec \prec p(z, \zeta) .
$$

In view of (2.1), we obtain

$$
q(z, \zeta) \prec \prec \frac{D_{m, \tau}^{\delta} f(z, \zeta)}{z}
$$

where

$$
q(z, \zeta)=\frac{1}{\lambda} z^{-\frac{1}{\lambda}} \int_{0}^{z} \psi(t, \zeta) t^{\frac{1}{\lambda}-1} d t
$$

is convex and it is the best subordinant.

If we combine the results of Theorem 2.1 and Theorem 2.2, we obtain the following strong differential "sandwich theorem".

Theorem 2.3. Let $\psi_{1}(z, \zeta)$ and $\psi_{2}(z, \zeta)$ be convex functions in $U \times \bar{U}$ with $\psi_{1}(0, \zeta)=\psi_{2}(0, \zeta)=1$ for every $\zeta \in \bar{U}$ and $\lambda>0$. If $f \in \mathcal{M}\left(\lambda, \delta, m, j, \tau ; \psi_{1}\right) \cap$ $\mathcal{N}\left(\lambda, \delta, m, j, \tau ; \psi_{2}\right), \frac{D_{m, \tau}^{\delta} f(z, \zeta)}{z} \in \mathcal{H}[1,1, \zeta] \cap Q_{\zeta}$ and

$$
\frac{1}{z}\left[\left(1-\frac{\lambda}{C_{j}^{m}(\tau)}\right) D_{m, \tau}^{\delta} f(z, \zeta)+\frac{\lambda}{C_{j}^{m}(\tau)} D_{m, \tau}^{\delta+1} f(z, \zeta)\right]
$$

is univalent in $U \times \bar{U}$, then

$$
f \in \mathcal{M}\left(0, \delta, m, j, \tau ; q_{1}\right) \cap \mathcal{N}\left(0, \delta, m, j, \tau ; q_{2}\right),
$$

where

$$
q_{1}(z, \zeta)=\frac{1}{\lambda} z^{-\frac{1}{\lambda}} \int_{0}^{z} \psi_{1}(t, \zeta) t^{\frac{1}{\lambda}-1} d t
$$

and

$$
q_{2}(z, \zeta)=\frac{1}{\lambda} z^{-\frac{1}{\lambda}} \int_{0}^{z} \psi_{2}(t, \zeta) t^{\frac{1}{\lambda}-1} d t
$$

The functions $q_{1}$ and $q_{2}$ are convex. 
Theorem 2.4. Let $\psi(z, \zeta)$ be a convex function in $U \times \bar{U}$ with $\psi(0, \zeta)=1$ for every $\zeta \in \bar{U}$ and

$$
G(z, \zeta)=\frac{\varepsilon+2}{z^{\varepsilon+1}} \int_{0}^{z} t^{\varepsilon} f(t, \zeta) d t,(z \in U, \zeta \in \bar{U}, \operatorname{Re}(\varepsilon)>-2)
$$

If $f \in \mathcal{M}(1, \delta, m, j, \tau ; \psi)$, then there exists a convex function $q(z, \zeta)$ such that $q(z, \zeta) \prec \prec \psi(z, \zeta)$ and $G \in \mathcal{M}(1, \delta, m, j, \tau ; q)$.

Proof. Suppose that

$$
p(z, \zeta)=\left(D_{m, \tau}^{\delta} G(z, \zeta)\right)_{z}^{\prime}, \quad(z \in U, \zeta \in \bar{U})
$$

Then, $p \in \mathcal{H}[1,1, \zeta]$.

From (2.3), we have

$$
z^{\varepsilon+1} G(z, \zeta)=(\varepsilon+2) \int_{0}^{z} t^{\varepsilon} f(t, \zeta) d t
$$

Differentiating both sides of (2.5) with respect to $z$, we get

$$
(\varepsilon+2) f(z, \zeta)=(\varepsilon+1) G(z, \zeta)+z G_{z}^{\prime}(z, \zeta)
$$

and

$$
(\varepsilon+2) D_{m, \tau}^{\delta} f(z, \zeta)=(\varepsilon+1) D_{m, \tau}^{\delta} G(z, \zeta)+z\left(D_{m, \tau}^{\delta} G(z, \zeta)\right)_{z}^{\prime}
$$

Differentiating the last relation with respect to $z$, we have

$$
\left(D_{m, \tau}^{\delta} f(z, \zeta)\right)_{z}^{\prime}=\left(D_{m, \tau}^{\delta} G(z, \zeta)\right)_{z}^{\prime}+\frac{1}{\varepsilon+2}\left(D_{m, \tau}^{\delta} G(z, \zeta)\right)_{z^{2}}^{\prime \prime}
$$

Since $f \in \mathcal{M}(1, \delta, m, j, \tau ; \psi)$, then we have

$$
\frac{1}{C_{j}^{m}(\tau) z}\left[D_{m, \tau}^{\delta+1} f(z, \zeta)-\left(1-C_{j}^{m}(\tau)\right) D_{m, \tau}^{\delta} f(z, \zeta)\right] \prec \prec \psi(z, \zeta)
$$

Now, from (1.5), (2.7) is equivalent to

$$
\left(D_{m, \tau}^{\delta} f(z, \zeta)\right)_{z}^{\prime} \prec \prec \psi(z, \zeta) .
$$


From (2.6) and (2.8), we get

$$
\left(D_{m, \tau}^{\delta} G(z, \zeta)\right)_{z}^{\prime}+\frac{1}{\varepsilon+2}\left(D_{m, \tau}^{\delta} G(z, \zeta)\right)_{z^{2}}^{\prime \prime} \prec \prec \psi(z, \zeta)
$$

Replacing (2.4) in (2.9), we obtain

$$
p(z, \zeta)+\frac{1}{\varepsilon+2} z p_{z}^{\prime}(z, \zeta) \prec \prec \psi(z, \zeta) .
$$

An application of Lemma 1.1 with $\mu=\varepsilon+2$, yields

$$
p(z, \zeta) \prec \prec q(z, \zeta) \prec \prec \psi(z, \zeta) .
$$

By using (2.4), we obtain

$$
\left(D_{m, \tau}^{\delta} G(z, \zeta)\right)_{z}^{\prime} \prec \prec q(z, \zeta) \prec \prec \psi(z, \zeta),
$$

where

$$
q(z, \zeta)=(\varepsilon+2) z^{-(\varepsilon+2)} \int_{0}^{z} \psi(t, \zeta) t^{\varepsilon+1} d t
$$

is convex and it is the best dominant.

Theorem 2.5. Let $\psi(z, \zeta)$ be a convex function in $U \times \bar{U}$ with $\psi(0, \zeta)=1$ for every $\zeta \in \bar{U}$ and $G(z, \zeta)$ is given by (2.3). If $f \in \mathcal{N}(1, \delta, m, j, \tau ; \psi)$, $\left(D_{m, \tau}^{\delta} G(z, \zeta)\right)_{z}^{\prime} \in \mathcal{H}[1,1, \zeta] \cap Q_{\zeta}$ and

$$
\frac{1}{C_{j}^{m}(\tau) z}\left[D_{m, \tau}^{\delta+1} f(z, \zeta)-\left(1-C_{j}^{m}(\tau)\right) D_{m, \tau}^{\delta} f(z, \zeta)\right]
$$

is univalent in $U \times \bar{U}$, then there exists a convex function $q(z, \zeta)$ such that $G \in \mathcal{N}(1, \delta, m, j, \tau ; q)$.

Proof. Suppose that the function $p(z, \zeta)$ be defined by (2.4). It is evident that $p \in \mathcal{H}[1,1, \zeta] \cap Q_{\zeta}$. After a short calculation and considering $f \in \mathcal{N}(1, \delta, m, j, \tau ; \psi)$, we can conclude that

$$
\psi(z, \zeta) \prec \prec p(z, \zeta)+\frac{1}{\varepsilon+2} z p_{z}^{\prime}(z, \zeta)
$$


An application of Lemma 1.2 with $\mu=\varepsilon+2$, yields

$$
q(z, \zeta) \prec \prec p(z, \zeta) .
$$

By using (2.4), we obtain

$$
q(z, \zeta) \prec \prec\left(D_{m, \tau}^{\delta} G(z, \zeta)\right)_{z}^{\prime},
$$

where

$$
q(z, \zeta)=(\varepsilon+2) z^{-(\varepsilon+2)} \int_{0}^{z} \psi(t, \zeta) t^{\varepsilon+1} d t
$$

is convex and it is the best subordinant.

If we combine the results of Theorem 2.4 and Theorem 2.5, we obtain the following strong differential "sandwich theorem".

Theorem 2.6. Let $\psi_{1}(z, \zeta)$ and $\psi_{2}(z, \zeta)$ be convex functions in $U \times \bar{U}$ with $\psi_{1}(z, \zeta)=\psi_{2}(z, \zeta)=1$ for every $\zeta \in \bar{U}$ and $G(z, \zeta)$ is given by (2.3). If $f \in$ $\mathcal{M}\left(1, \delta, m, j, \tau ; \psi_{1}\right) \cap \mathcal{N}\left(1, \delta, m, j, \tau ; \psi_{2}\right),\left(D_{m, \tau}^{\delta} G(z, \zeta)\right)_{z}^{\prime} \in \mathcal{H}[1,1, \zeta] \cap Q_{\zeta}$ and

$$
\frac{1}{C_{j}^{m}(\tau) z}\left[D_{m, \tau}^{\delta+1} f(z, \zeta)-\left(1-C_{j}^{m}(\tau)\right) D_{m, \tau}^{\delta} f(z, \zeta)\right]
$$

is univalent in $U \times \bar{U}$, then

$$
f \in \mathcal{M}\left(1, \delta, m, j, \tau ; q_{1}\right) \cap \mathcal{N}\left(1, \delta, m, j, \tau ; q_{2}\right),
$$

where

$$
q_{1}(z, \zeta)=(\varepsilon+2) z^{-(\varepsilon+2)} \int_{0}^{z} \psi_{1}(t, \zeta) t^{\varepsilon+1} d t
$$

and

$$
q_{2}(z, \zeta)=(\varepsilon+2) z^{-(\varepsilon+2)} \int_{0}^{z} \psi_{2}(t, \zeta) t^{\varepsilon+1} d t
$$

The functions $q_{1}$ and $q_{2}$ are convex. 


\section{References}

[1] F. M. Al-Oboudi, On univalent functions defined by a generalized Sălăgean operator, Int. J. Math. Math. Sci. 27 (2004), 1429-1436. https://doi.org/10.1155/S0161171204108090

[2] B. A. Frasin, A new differential operator of analytic functions involving binomial series, Bol. Soc. Paran. Mat. 38(5) (2020), 205-213. https://doi.org/10.5269/bspm.v38i5.40188

[3] S. S. Miller and P. T. Mocanu, Differential Subordinations: Theory and Applications, Series on Monographs and Textbooks in Pure and Applied Mathematics, Vol. 225, Marcel Dekker Inc., New York and Basel, 2000.

[4] G. I. Oros, Strong differential superordination, Acta Univ. Apulensis 19(2009), 101-106.

[5] G. I. Oros and Gh. Oros, Strong differential subordination, Turk. J. Math. 33(3) (2009), 249-257.

[6] G. S. Salagean, Subclasses of univalent functions, Lecture Notes in Math. 1013, Springer Verlag, Berlin, 1983, pp. 362-372. https://doi.org/10.1007/BFb0066543

[7] F. Yousef, T. Al-Hawary and G. Murugusundaramoorthy, Fekete-Szegö functional problems for some subclasses of bi-univalent functions defined by Frasin differential operator, Afrika Matematika 30(3-4) (2019), 495-503.

https://doi.org/10.1007/s13370-019-00662-7 